Ann. Biol. anim. Bioch. Biophys., I965, 5 (2), 223-236.

\title{
COMPARAISON DE L'ACTION DE L'ACRYLATE DE SODIUM ET DE LA CHLORTÉTRACYCLINE SUR LA FLORE INTESTINALE DU PORC
}

\author{
M. C. MICHEL \\ Avec la collaboration technique de Simone Boche et Geneviève Hannequart \\ Laboratoire des Métabolismes, \\ Centre national de Recherches zootechniques, Jouy-en-Josas (Seine-et-Oise)
}

\section{SOMMAIRE}

I. In vitro l'acrylate de sodium, à la dose de I,5 p. I ooo, inhibe sélectivement la croissance d'Escherichia coli dans la flore complexe de l'intestin du porc; la croissance des lactobacilles n'est pas modifiée; la chlortétracycline (20 $\mathrm{mg} /$ litre) modifie peu la répartition de ces deux espèces.

2. Le catabolisme azoté de la flore (mesuré par la dégradation de l'urée, de la L-arginine et de la L-lysine) est inhibé au même taux par l'acrylate et la chlortétracycline (environ 90 p. Ioo en 6 heures).

3. La dégradation des glucides par la flore, hétérofermentaire chez le témoin (formation de $\mathrm{CO}_{2}$ et d'acides volatils), est orientée vers une production pratiquement exclusive d'acide lactique sous l'effet des deux inhibiteurs, mais la vitesse de formation de l'acide lactique est augmentée par l'acrylate et diminuée par la chlortétracycline.

4. Ces résultats peuvent rendre compte, du moins en partie, de la stimulation de croissance du porcelet produite par ces deux substances, par l'intermédiaire d'une meilleure utilisation de l'azote et de l'énergie de la ration.

L'acide acrylique, ou son sel de sodium $\left(\mathrm{CH}_{2}=\mathrm{CH}-\mathrm{CO}_{2} \mathrm{Na}\right)$ administré par voie orale, inhibe le développement des coliformes de la flore microbienne intestinale du poulet (SIEBURTH, Ig60-I96I). Cette observation offre une voie d'approche intéressante pour l'étude des relations entre l'hôte et sa flore intestinale. En effet, il est bien établi que certaines espèces microbiennes, présentes normalement dans l'intestin, sont susceptibles d'exercer une influence défavorable sur la croissance de l'hôte (notamment dans le cas du poulet). Cette action dépressive peut être annulée par divers antibiotiques (voir la revue de Françors (I962), et FrançoIs et MIchEL, (Ig63)). 
L'observation de SIEBURTH pose la question suivante : l'élimination des coliformes est-elle la seule cause de la stimulation de croissance observée chez le poulet (Whi'TE-STEVENS et al., I96I) ou bien cette action s'étend-elle à d'autres espèces microbiennes, et dans ce cas comment l'acrylate agit-il sur la flore?

Chez le porcelet de 5 à 9 semaines, l'acrylate et l'oxytétracycline réduisent notablement l'excrétion fécale d'ammoniac (MIchel et al., Ig64); ce ne peut être đû à la seule élimination des coliformes, puisque contrairement à l'acrylate, les tétracyclines exercent une action favorable sur le développement intestinal d'Escherichia coli, par augmentation du nombre de ces derniers en présence de quantités inchangées de lactobacilles, streptocoques, etc. (RAIbaud, I958). Afin de connaître le mécanisme de ces effets contradictoires, le but de cette étude est de comparer l'influence de l'acrylate et de la chlortétracycline sur la flore intestinale du porc.

\section{MÉTHODES}

La flore intestinale est incubée en présence du substrat choisi, dans des conditions aussi proches que possible de celles qui existent dans l'intestin (température, concentration respective des germes et du substrat, durée d'incubation; valeurs du $\mathrm{pH}$, lequel conditionne la synthèse des enzymes adaptifs et leur activité (GALE, I946) et du Eh, qui détermine le sens des réactions enzymatiques WURMSER, 1935)).

\section{Isolement de la flore intestinale}

Dès l'abattage de l'animal (porcs de 90 à $100 \mathrm{~kg}$ ), le contenu du cæcum est prélevé et homogénéisé. On pèse $100 \mathrm{~g}$ de cette suspension et on mélange les prélèvements effectués sur to animaux. Le prélèvement moyen est dilué au $\mathrm{I} / 2(\mathrm{v} / \mathrm{v})$, par de l'eau distillée, puis après homogénéisation à l'agitateur, filtré sur une toile de nylon très fine, de manière à éliminer la majeure partie des particules alimentaires. Le filtrat est conservé à $+4^{\circ} \mathrm{C}$ jusqu'au moment de l'emploi -4 jours au maximum -- (une température inférieure à $\circ^{\circ} \mathrm{C}$ inactive les actions enzymatiques).

Ce procédé simple permet d'obtenir une suspension microbienne dont l'activité n'est pas modifiée, contrairement à ce qui se passe lorsque les microbes sont centrifugés et lavés (LEWIS et EMERY, 1962).

\section{Métabolisme en phase proliférante}

Avec I à ıo p. Ioo (v/v) de suspension microbienne, on inocule le bouillon suivant :

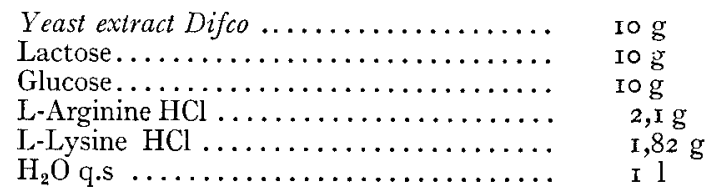

Autoclavage $15 \mathrm{mn}$ à $\mathrm{I} 20^{\circ} \mathrm{C}$.

Le pH est ajusté à 6,8 (mesuré à l'électrode de verre), au début de l'incubation et maintenu à cette valeur pendant la phase proliférante, par addition de soude normale.

L'incubation est effectuée au bain-marie à $3^{8^{\circ} \mathrm{C}}$ sous agitation magnétique. Dans ces conditions, le potentiel d'oxydoréduction s'établit aux environs de - $200 \mathrm{mV}$, valeur voisine de celle trouvée dans l'intestin.

Les inhibiteurs sont ajoutés à la dose de $1,5 \mathrm{~g} / \mathrm{l}$ (acrylate de sodium) ou $20 \mathrm{mg} / \mathrm{l}$ (chlortétracycline). 
Pour les essais en phase stationnaire.

La suspension est simplement diluée (v/v) dans le substrat (acides aminés $\mathrm{M} / 25$, glucose $40 \mathrm{~g} / \mathrm{l}$ ). L'incubation est effectuée comme précédemment.

La suspension contient une faible quantité d'acides aminés, de peptides, de $\mathrm{NH}_{3}$ et de bases aminées. Ces valeurs sont déterminées par l'analyse du témoin et déduites éventuellement des résultats des lots expérimentaux.

\section{ANALYSES}

A divers intervalles de temps, généralement toutes les deux heures, on prélève $20 \mathrm{ml}$ de suspension afin d'effectuer les mesures suivantes :

Io Densité optique à $570 \mathrm{~m} \mu$, après dilution au $\mathrm{I} / 20$ dans de l'eau distillée.

Pour les déterminations suivantes, le prélèvement est porté $5 \mathrm{mn}$ à $100^{\circ} \mathrm{C}$, au bain-marie, puis après retroidissement, centrifugé $25 \mathrm{mn}$ à $9000 \mathrm{~g}$. Le surnageant est conservé à $-{ }^{\circ} 5^{\circ}$ jusqu'au moment du dosage.

$2^{\circ}$ Glucose et lactose (Mendel, 1954).

$3^{\circ}$ Acide lactique (Barker et Summerson, I94I).

$4^{\circ}$ Azote ammoniacal (Conway, 1957).

$5^{\circ}$ Azote alpha-aminé (Michel, I96i a). 1965).

$6^{\circ}$ Acides aminés basiques et amines par chromatographie sur résine cationique faible (MICHEL,

$7^{\circ}$ Les numérations microbiennes sont effectuées, après dilutions décimales, sur les milieux suivants :

"Violet red bile agar "Difco pour les coliformes.

Milieu décrit par Raibaud (196I) pour les bacilles lactiques.

\section{RÉSULTATS}

\section{$I^{0}$ Croissance de la flore complexe}

L'acrylate et la chlortétracycline ralentissent la vitesse de croissance de la culture et diminuent légèrement la quantité de germes formés à la fin de la phase de croissance. Ils provoquent en outre, une autolyse des germes plus rapide, à la fin de la croissance que chez le témoin (fig. I).

\section{$2^{\circ}$ Résistance des germes}

L'acrylate et la chlortétracycline exercent une action très différente sur la croissance des lactobacilles et des coliformes dans la flore complexe (tabl. I).

Les modifications observées peuvent être dues, d'une part au développement préférentiel des espèces les moins sensibles, et d'autre part à l'apparition des mutants résistants.

En ce qui concerne la résistance à l'acrylate, aucune observation in vivo ne semble avoir été effectuée. En première analyse les modifications apportées par cette substance dans la composition de la flore peuvent être dues en grande partie à la plus grande sensibilité des coliformes (tabl. 2).

Ces résultats sont en accord avec les observations de SiEbURTh (I96I), en ce qui concerne l'inhibition de la croissance des coliformes par l'acrylate chez le poulet, 


\section{TABLEAU I}

Influence de lacrylate et de la chlortétracycline sur la croissance microbienne

\begin{tabular}{l|c|c|c}
\hline \hline & Densité optique * & $\begin{array}{c}\text { Logarithme du nombre } \\
\text { des colibacilles }\end{array}$ & $\begin{array}{c}\text { Logarithme du nombre } \\
\text { des lactobacilles }\end{array}$ \\
\hline Témoin $\ldots \ldots \ldots \ldots \ldots \ldots \ldots \ldots \ldots$ & 6,1 & 6,8 & 9,0 \\
\hline Acrylate 1,5 p. $1000 \ldots \ldots \ldots \ldots \ldots$ & 5,1 & $4,7 * *$ & 9,5 \\
\hline Chlortétracycline $20 \mathrm{mg} / \mathrm{ml} \ldots \ldots \ldots$ & 5,9 & 8,5 & 9,0 \\
\hline
\end{tabular}

Culture de $22 \mathrm{~h}$ - ensemencée avec 1 p. 100 de suspension (contenu cæcal au 1/2)

* L'unité de densité optique correspond environ à $1 \mathrm{~g}$ de poids sec par litre

** Ce nombre est celui existant dans la culture au début de l'incubation soit $10^{6}$ dans le contenu du cæcum et dilution au $1 / 200$ dans le bouillon.

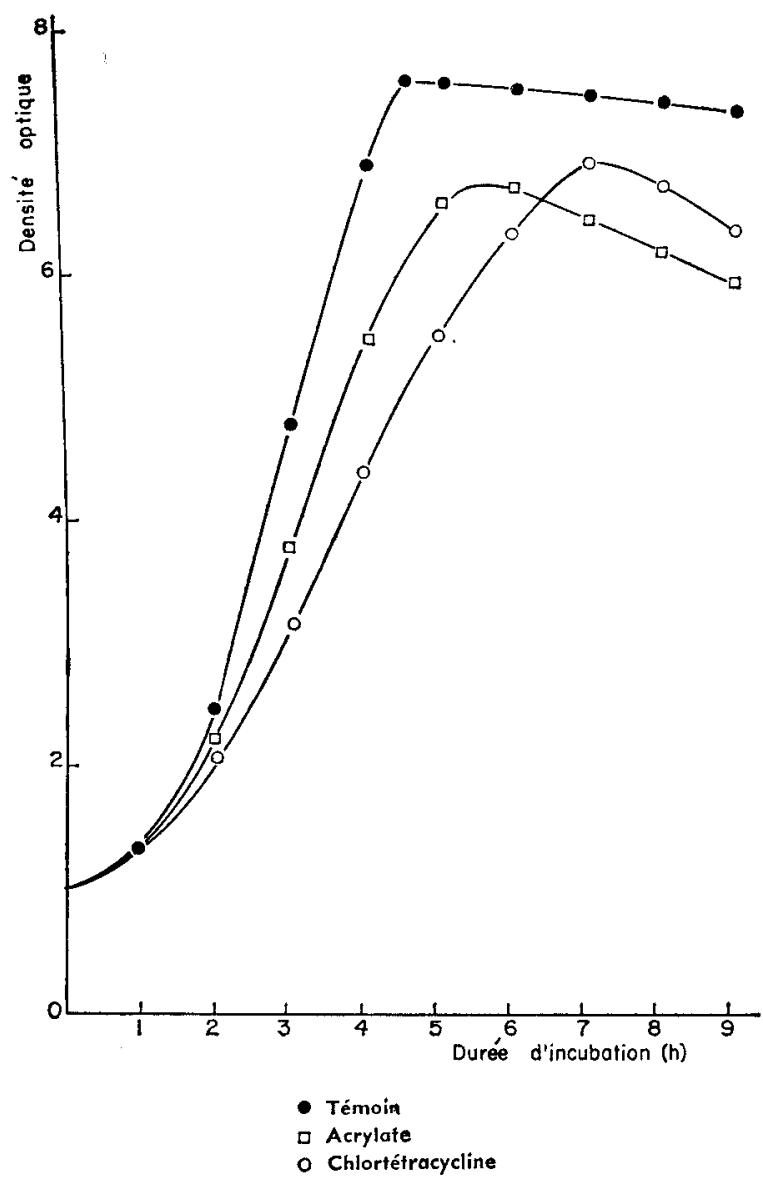

FIG. I. - Action comparée de l'acrylate et de la chlortétracycline sur la croissance microbienne 


\section{TABLEAU 2}

Influence de l'acrylate sur la croissance d'espèces isolées

\begin{tabular}{|c|c|c|}
\hline & \multicolumn{2}{|c|}{ Densité optique moyenne } \\
\hline & Acrylate & Témoin \\
\hline Lactobacilles homofermentaires 10 souches & 1,510 & $1,6: 0$ \\
\hline Escherichia Coli 14 souches $\ldots \ldots \ldots \ldots$ & 0,080 & 0,558 \\
\hline
\end{tabular}

(Bouillon ensemencé avec une culture de $2 \dot{x}$ h $(1$ p. 100) $-2 x$ h d'incubation).

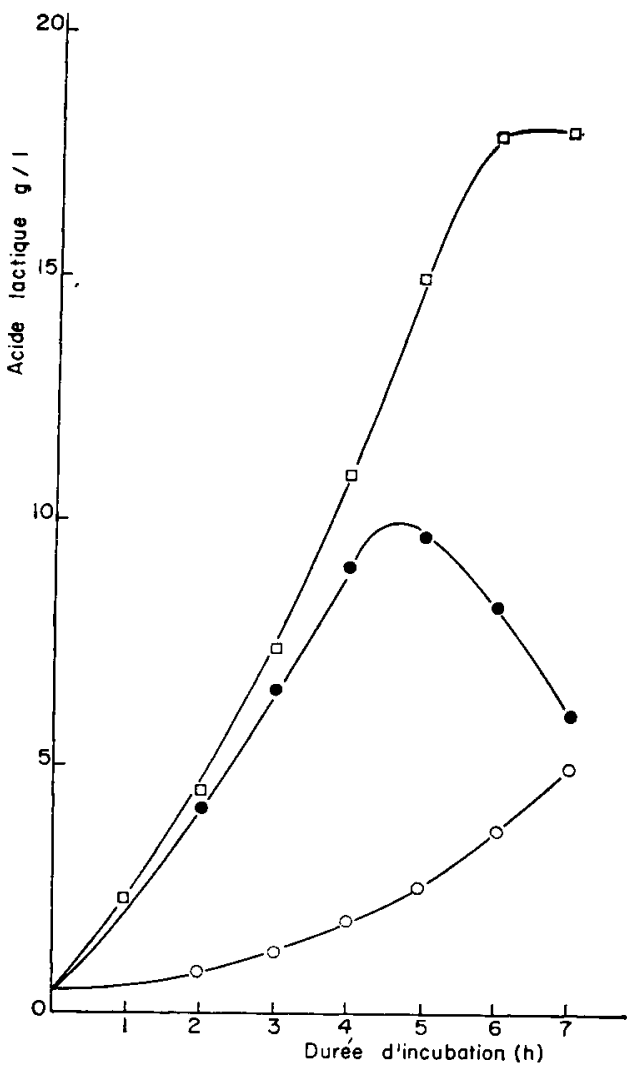

- Témoin

口 Acrylate

- Chlortétracycline

FIG. 2. - Formation d'acide lactique à parlir du glucose 
et de RAIBAud (I958), pour ce qui est de l'action de la chlortétracycline sur la croissance des coliformes et des lactobacilles dans l'intestin du porc et du rat, à savoir, l'augmentation du rapport coliformes sur lactobacilles.

\section{$3^{\circ}$ Métabolisme du glucose}

Un autre aspect de ces effets sur la croissance des différentes espèces se manifeste au cours de la glycolyse. L'acrylate augmente la production d'acide lactique, qui, contrairement à ce qu'on observe dans la culture témoin, n'est pas dégradé pendant la phase de croissance. La chlortétracycline inhibe à la fois la dégradation de la source carbonée et celle de 1'acide lactique formé, ainsi que nous l'avons démontré précédemment (MICHEL, Ig6I c) (fig. 2).

Rapportés au poids sec microbien, les résultats, après 7 heures d'incubation, sont exprimés dans le tableau 3.

TABLEAU 3

Influence de l'acrylate et de la chlortétracycline sur la glycolyse.

\begin{tabular}{|c|c|c|c|c|c|c|}
\hline & $\begin{array}{l}\text { Glucose } \\
\text { dégradé }\end{array}$ & $\begin{array}{l}\text { Acide } \\
\text { lactique } \\
\text { formé }\end{array}$ & $\begin{array}{l}\text { Poids sec } \\
\text { microbien }\end{array}$ & $\begin{array}{c}\text { Iactate / } \\
\text { Glucose } \\
\text { P. } 100\end{array}$ & \begin{tabular}{|} 
g de glucose \\
dégradé/g de \\
poids sec \\
microbien
\end{tabular} & $\begin{array}{l}g \text { de lactate } \\
\text { formé } / g \text { de } \\
\text { poids sec } \\
\text { microbien }\end{array}$ \\
\hline Témoin $\ldots . . . \ldots$. & $20 \mathrm{~g}$ & $6 \mathrm{~g}$ & 7,3 & 30 & 2,74 & 0,82 \\
\hline Acrylate...$\ldots \ldots$ & $20 \mathrm{~g}$ & $18 \mathrm{~g}$ & 6,5 & 90 & 3,18 & 2,77 \\
\hline Chlortétracycline ... & $5,5 \mathrm{gr}$ & $5 \mathrm{~g}$ & 6,8 & 91 & 0,81 & 0,73 \\
\hline
\end{tabular}

On voit que l'action des deux substances, bien que très différente dans sa cinétique, aboutit aux mêmes résultats, c'est-à-dire une fermentation qui s'apparente à celle des lactobacilles homofermentaires. On sait que ces derniers dégradent les glucides avec une production presque exclusive d'acide lactique, alors que des espèces sous-dominantes de la flore (lactobacilles hétérofermentaires, Streptocoques coliformes, Clostridia), produisent divers acides organiques : (pyruvique, acétique, butyrique et lactique) du $\mathrm{CO}_{2}$, des alcools, etc. (BERGEY's Manual, I953). L'hétérofermentation observée dans les cultures témoins (Mrches, I96I c) explique le faible rendement en acide lactique. Il résulte de ces modifications, produites par la chlor tétracycline et l'acrylate, une épargne énergétique. On sait en effet que la formation du lactate libère moins d'énergie que la métabolisation complète du glucose (KREBS, I954).

\section{4) Métabolisme azoté}

Ia dégradation des sucres conditionne le métabolisme azoté : en particulier, celui de l'ammoniac, pour lequel on distingue deux phases nettement distinctes. Pendant la phase proliférante, on observe une diminution de la teneur en $\mathrm{NH}_{3}$ et 
acides aminés du milieu. L'activité catabolique des germes est faible. Dès que la source carbonée a disparu (après 5 à 6 heures d'incubation), 1'activité catabolique à l'égard des stubstances azotées atteint son maximum, ainsi que le montre la libération de $\mathrm{NH}_{3}$. L'acrylate et la chlortétracycline inhibent l'utilisation de $\mathrm{NH}_{3}$ par la flore proliférante et la formation de cette substance pendant l'autolyse (fig. 3).

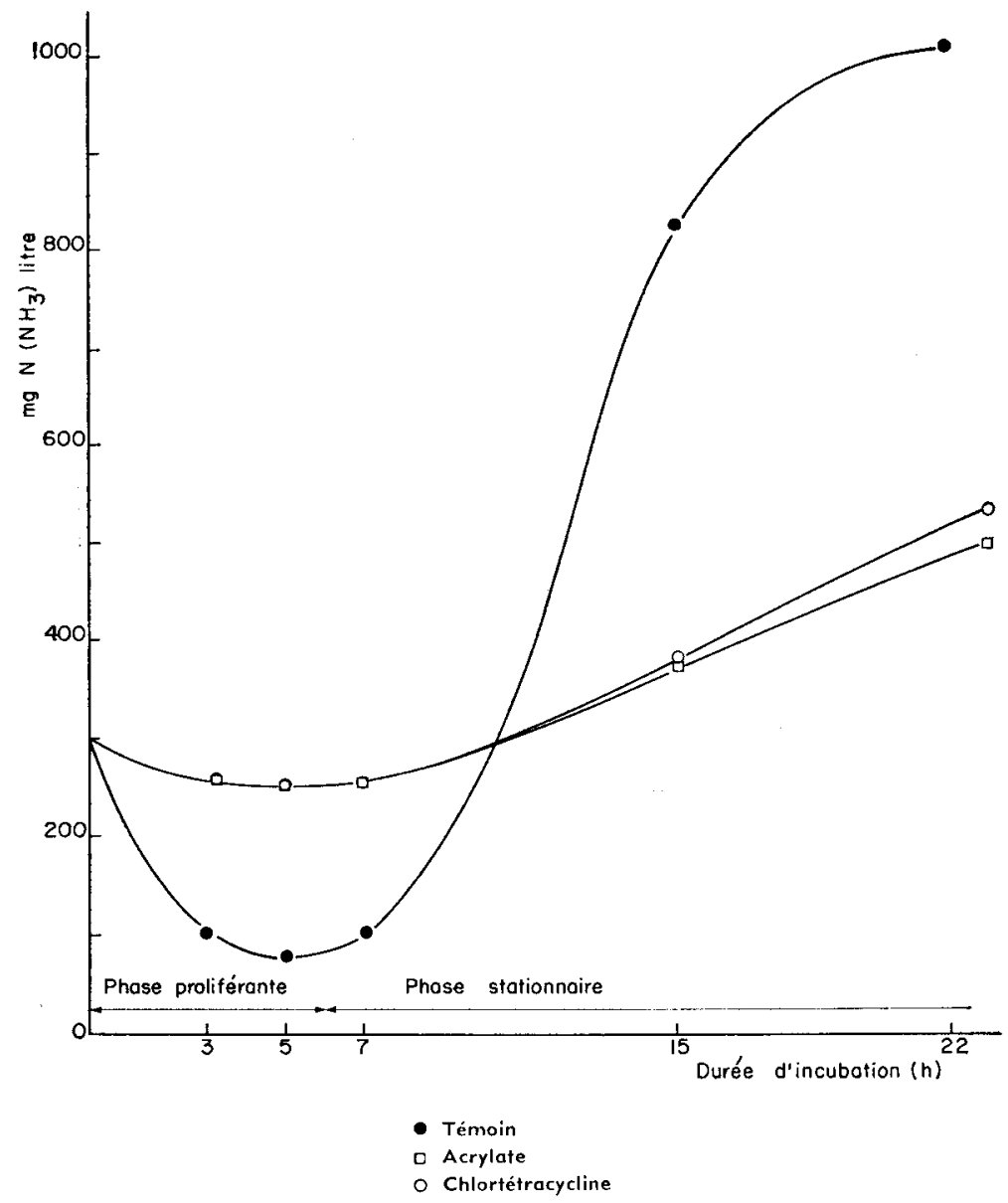

FIG. 3. - Métabolisme de l'ammoniac au cours des différentes phases de la croissance microbienne

Dans le tractus intestinal du porc, la présence des glucides solubles dans l'estomac et l'intestin grêle, est associée à une teneur faible en ammoniac et à une activité catabolique faible. Dès que la source carbonée a disparu, à partir du cæcum, le taux de $\mathrm{NH}_{3}$ et l'activité microbienne augmentent de manière considérable (fig. 4).

\section{Métabolisme de l'urée}

Bien que le taux de $\mathrm{NH}_{3}$ puisse s'abaisser pendant la glycolyse, diverses substances azotées peuvent être dégradées; l'urée en particulier. Cette substance est amenée au niveau intestinal par la salive et le sang. Divers microbes intestinaux 
(Klebsellia - Aerobacter - Proteus - Streptocoques, etc.) 1'hydrolysent avec formation de $\mathrm{NH}_{3}$ et $\mathrm{CO}_{2}$.

In vitro, la chlortétracycline et l'acrylate inhibent l'action uréasique d'une culture proliférante. Puisque ces substances sont sans action sur l'uréase microbienne,

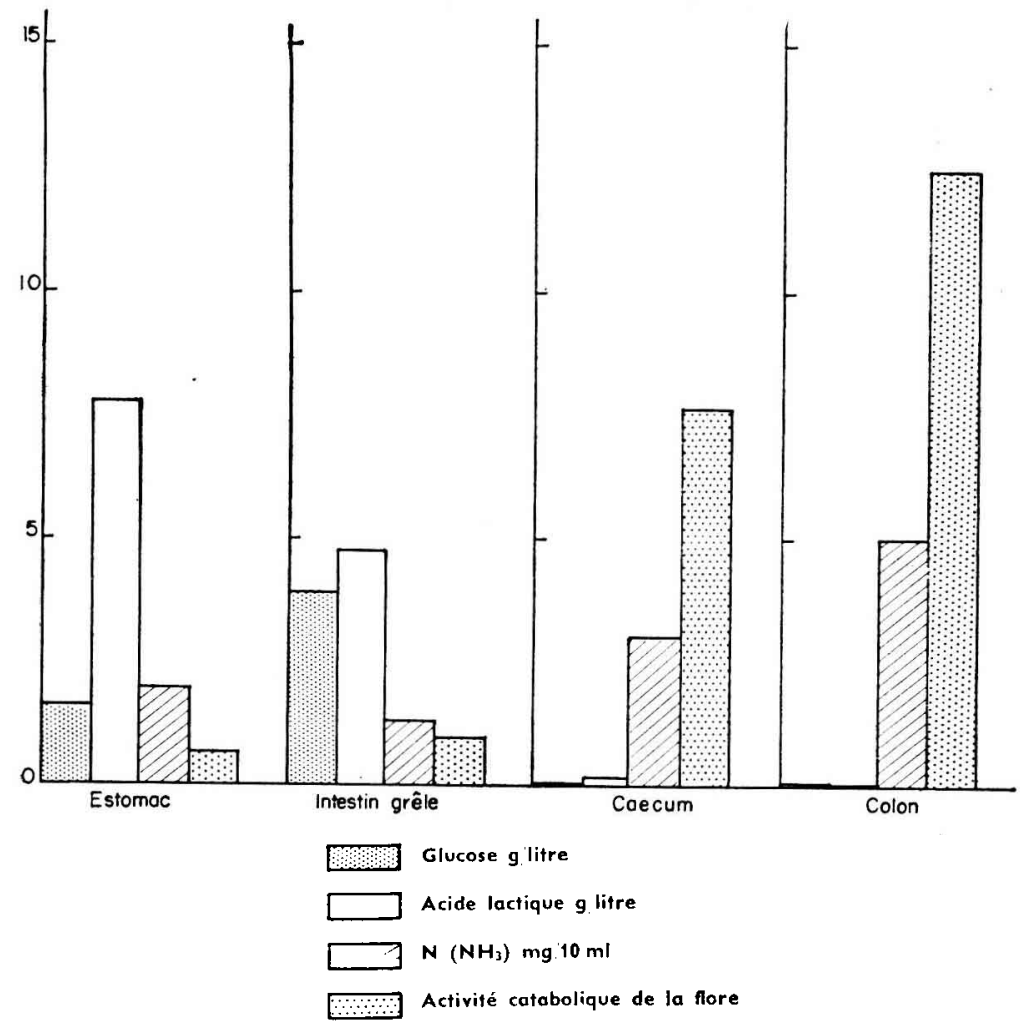

FIG. 4. - Evolution dans le tractus digestif du porc de la teneur en glucose, acide lactique et asote ammoniacal, en liaison avec l'activité catabolique de la flore (exprimée en millimolécules d'arginine dégradées par heure et par litre de contenu)

TABLEAU 4

Dégradation de l'urée par une culture proliférante

\begin{tabular}{|c|c|c|c|}
\hline & \multicolumn{3}{|c|}{$\operatorname{mg} \times\left(\mathrm{NH}_{3}\right)$ formé par $\mathrm{g}$ de poids $\mathrm{sec}$} \\
\hline & $2 \mathrm{~h}$ & $4 h$ & $6 \mathrm{~h}$ \\
\hline Témoin .................. & 150 & 210 & 378 \\
\hline Acrylate . . . . . . . . . . . & 108 & $9 f_{j}$ & 128 \\
\hline Chlortètracycline ........... & 108 & 95 & 126 \\
\hline
\end{tabular}

(1) L'urée, à la dose de 3 p. 1000 est ajoutée au bouillon en même temps que l'inoculum. 
en phase stationnaire, cela suggère que c'est la croissance des espèces uréase + , ou la synthèse de l'uréase, qui est bloquée. Rapportée au poids sec microbien, la dégradation est constante pendant 5 heures et correspond à l'activité de l'inoculum (tabl. 4).

L'action des deux inhibiteurs semble procéder du même mécanisme. I ls'agit de l'inhibition d'autres germes que Escherichia coli, puisque sur un grand nombre de souches de cette espèce isolées par DIckixson (I96I), alicune ne dégradait l'urée.

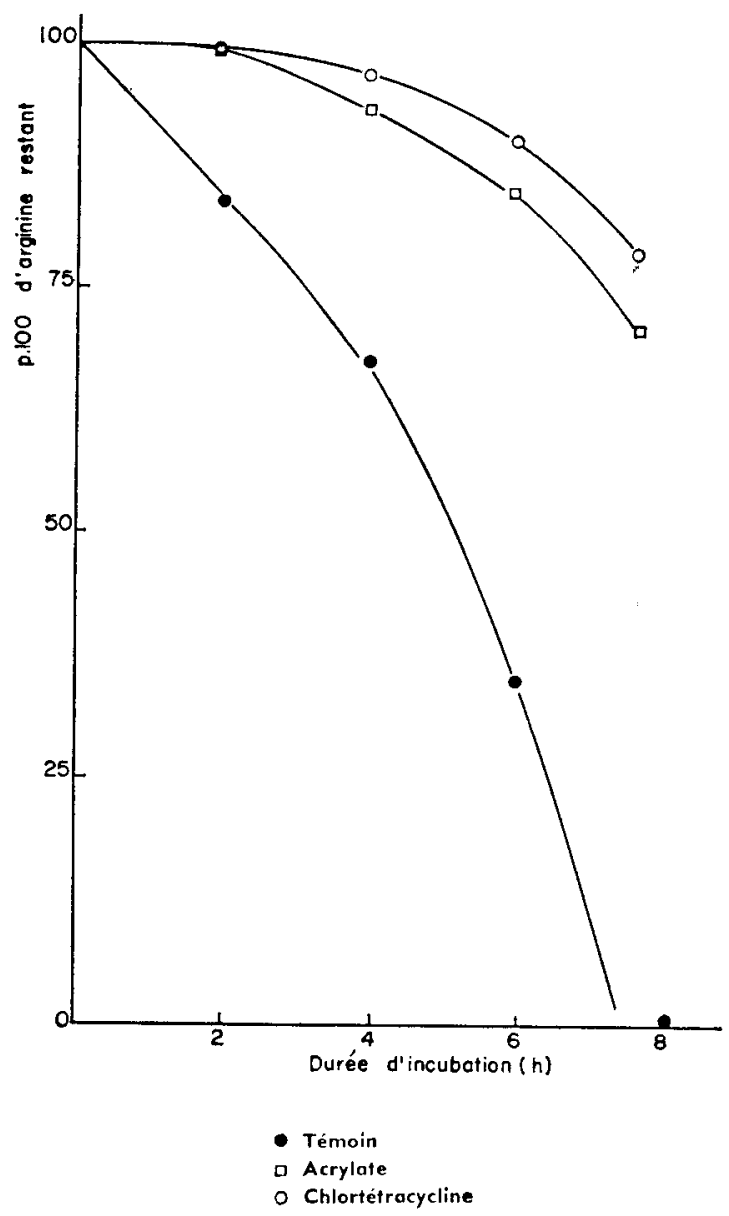

FIG. 5. - Dégradation de la L-arginine par la flore du caecum du porc

\section{Dégradation de la lysine et de l'arginine}

De même que 1'urée, ces deux acides aminés basiques sont rapidement dégradés par la flore en phase proliférante (ce catabolisme est mesuré par dosage chromatographique au cours de l'incubation). L'acrylate et la chlortétracycline inhibent cette dégradation (fig. 5 et 6 et tabl. 5). 
Il résulte de cette inhibition une moindre formation des diamines qui sont les produits finaux de la réaction: putrescine pour l'arginine et cadavérine pour la lysine. Ces résultats sont en accord avec ceux de LARSoN et HILL (I960), relatifs à l'inhibition de la formation d'amines dans l'iléon du jeune porc ingérant de la chlortétracycline, et ceux de MELNYKOWYCZ (I956), qui a montré que la synthèse adaptive de certaines amino-acides décarboxylases de la flore intestinale du rat était bloquée par cet antibiotique.

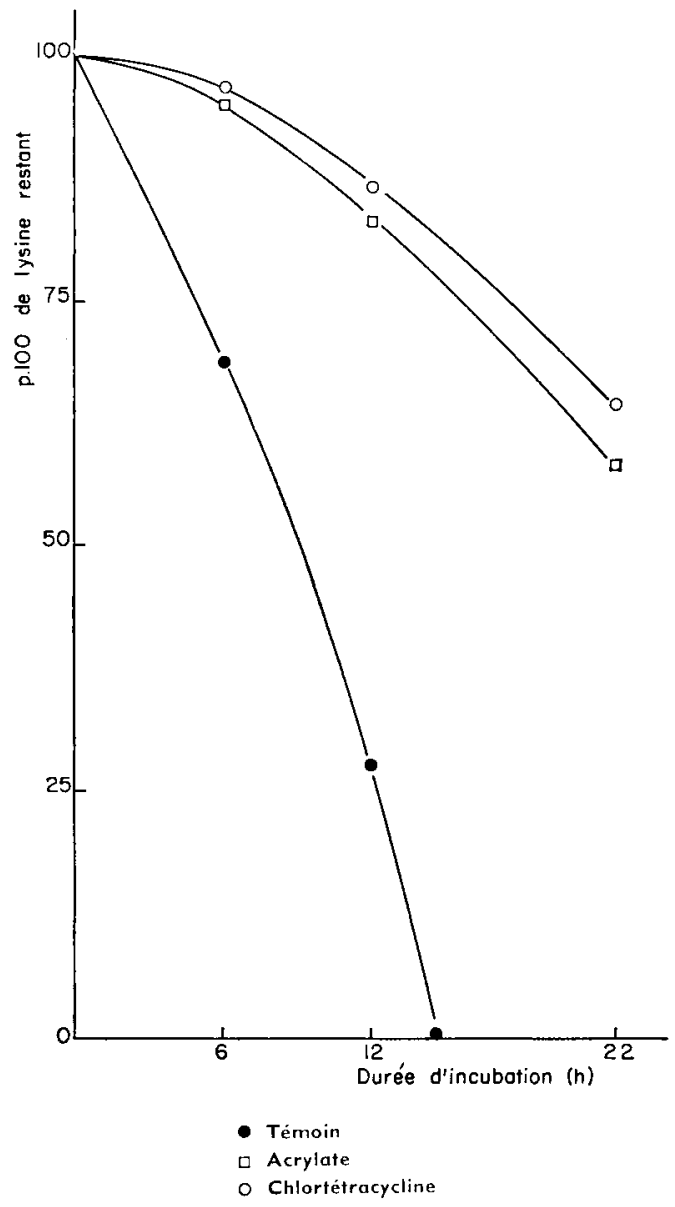

Fig. 6. - Dégradation de la L-lysine par la fore du caecum du porc

\section{DISCUSSION}

In vitro, et aux doses utilisées dans la pratique de l'alimentation animale, la chlortétracycline et l'acrylate exercent le même effet inhibiteur sur la dégradation des substances azotées par la flore intestinale du porc. En ce qui concerne la dégra- 
dation du glucose, seule la chlortétracycline ralentit le catabolisme de cette substance ainsi que celui de l'acide lactique formé. L'acrylate, qui n'inhibe pas la glycolyse, déplace également l'équilibre vers la production presque exclusive d'acide lactique, vraisemblablement par son action bactériostatique plus élevée à l'encontre des germes hétérofermentaires. Dans quelle mesure ces modifications peuvent-elles avoir lieu chez l'animal vivant et influer sur son métabolisme? En ce qui concerne la chlortétracycline, celle-ci, ingérée par le porc, abaisse notablement le taux d'ammoniac du sang de la veine porte (FrançoIs et MichEL, I960). Elle inhibe également la formation d'amines dans l'intestin grêle du porcelet, ainsi que l'activité décarboxylante du contenu iléal (mesurée in vitro); bien que le nombre total des coliformes ne soit pas modifié (LARSON et HILL, I960).

TABLEAU 5

Influence de l'acrylate et de la chlortétracycline sur la dégradation de la lysine et de l'arginine

\begin{tabular}{c|c|c|c||c|c|c}
\hline \hline & $\begin{array}{c}\mu M \\
\text { d'arginine } \\
\text { dégradée } / \mathrm{ml}\end{array}$ & $\begin{array}{c}\mu \mathrm{M} / \text { heure } / g \\
\text { poids sec }\end{array}$ & $\begin{array}{c}\text { Pourcentage } \\
\text { par rapport } \\
\text { au témoin }\end{array}$ & $\begin{array}{c}\mu M \text { de lysine } \\
\text { dégradée } / \mathrm{ml}\end{array}$ & $\begin{array}{c}\mu \mathrm{M} / \text { heure } / g \\
\text { poids sec }\end{array}$ & $\begin{array}{c}\text { Pourcentage } \\
\text { par rapport } \\
\text { an témoin } \\
.\end{array}$ \\
\hline Témoin ........ & 10,5 & 0,344 & 100 & 7,6 & 0,141 & 100 \\
\hline Acrylate ........ & 2 & 0,043 & 12,5 & 0,75 & 0,016 & 11,3 \\
\hline Chlortétracycline $\ldots$ & 3 & 0,062 & 18 & 1,0 & 0,020 & 14,2 \\
\hline \hline
\end{tabular}

Cette inhibition du catabolisme azoté peut expliquer les résultats de ZELTER - (rg6r) et de DelorT-LAVAL (1963) qui observaient une augmentation de la rétention azotée chez le porc par la chlortétracycline.

D'autres résultats vont dans le même sens. Ainsi, Michę et al. (r964) ont observé que l'acrylate et l'oxytétracycline, dont les propriétés sont voisines de celles de la chlortétracycline, produisaient une stimulation de croissance et une augmentation de l'efficacité alimentaire chez le porcelet. Une corrélation inverse avait pu être établie entre l'activité de la flore (mesurée par le taux de $\mathrm{NH}_{3}$ et des amines dans les fèces) et la stimulation de croissance. En effet, cette activité était presque nulle jusqu'à 5 semaines, date du sevrage, et s'élevait rapidement ensuite jusqu'à 9 semaines. Pendant cette dernière période, l'action favorable sur la croissance était associée à une nette diminution de 1'ammoniac excrété.

En outre, l'inhibition de l'activité uréasique de la flore peut être une des causes de cette diminution et rendre compte, dans une certaine mesure, de la stimulation de croissance observée. En effet, d'après les travaux de DANG et VisEK (I960), de VISEK (Ig62), les animaux dont l'activité uréasique intestinale est inhibée (par voie immunologique), présentent un abaissement significatif de l'indice de consommation (rats), ou une stimulation de leur croissance (poulets). Il reste à déterminer l'activité uréasique du contenu intestinal des animaux ayant ou non ingéré des antibiotiques, afin de vérifier si l'inhibition observée in vitro s'effectue également in vivo. 
In vitro, l'inhibition du catabolisme azoté est associée à une orientation de l'activité fermentaire de la flore vers une production presque exclusive d'acide lactique. Or, nous avons démontré précédemment que diverses espèces microbiennes (Streptocoques, entérobactéries, Clostridia, etc.) qui sont hétérofermentaires, possèdent également une activité catabolique élevée à l'égard des substances azotées (MrcHEL, I96I $d$ ). Ceci peut être rapproché du rôle favorable d'une flore "lactique " au niveau intestinal qui, bien que controversé, a été démontré dans plusieurs cas. Chez le nourrisson humain alimenté au lait maternel, les troubles digestifs sont plus rares que chez les sujets qui reçoivent du lait de vache. Or, la flore intestinale des premiers est constituée de Lactobacillus bifidus, homofermentaire, en culture pratiquement pure, alors que celle des seconds comprend de nombreuses entérobactéries (GYöRGY, I957). Les souris Pathogen-free de Dubos, qui n'hébergent, à côté d'une flore de lactobacilles, que des quantités infimes de germes d'autres espèces, présentent une vitesse de croissance, une résistance aux endotoxines microbiennes, etc. de beaucoup supérieure à celle des animaux conventionnels (Dubos, Ig60).

Les résultats obtenus par RAIBAUD (I958) et par MASKA (Ig60), seraient contradictoires si on se bornait à considérer uniquement l'accroissement du nombre des coliformes, produits par la chlortétracycline dans l'intestin du porc, et non l'activité métabolique de la flore. Nos résultats qui confirment et étendent les observations de LaRson et HILL (I960) et de MELnikowycz et Johansson (I955), montrent que, bien que le nombre de germes soit peu modifié, leur catabolisme azoté est inhibé et leur catabolisme glucidique déplacé dans le sens d'une fermentation lactique. La croissance de souches mutantes résistantes à l'antibiotique, et dont le métabolisme est ralenti, pourrait expliquer ces différences.

En ce qui concerne l'acrylate, les différences observées avec la chlortétracycline, quant à leur mode d'action, laissent supposer que leurs effets sur la croissance pourraient être additifs. C'est ce qui ressort des travaux de WhITE-STEvENs et al. (rg62) sur le poulet.

Il reste à préciser, d'une part la nature des substances dépressives de la croissance : dérivés des substances azotées ou des glucides, et d'autre part la nature intime de 1'action de l'acrylate.

Reçu pour publication en février 1965.

\section{SUMMARY}

COMPARISON OF THE EFFECTS OF SODIUM ACRYLATE

AND CHLORTETRACYCLINE ON THE INTESTINAL FLORA OF THE PIG

I. In the complex flora of the intestine of the pig growth of Escherichia coli was inhibited selectively in vitro by 0.1 per cent sodium acrylate; there was little change in growth of lactobacilli. With $20 \mathrm{mg}$ chlortetracycline per litre there was little change in the distribution of these two species. For lactobacilli the logarithm of the number of bacteria per $\mathrm{ml}$ was 9.0 in controls, 9.5 with acrylate and 9.0 with chlortetracycline.

2. Nitrogen catabolism of the flora, measured by degradation of urea, L-lysine and L-arginine were inhibited to the same extent by acrylate and chlortetracycline, about go per cent in $6 \mathrm{~h}$. 
3. The degradation of carbohydrates by the flora, by fermentation of mixed types with the formation of $\mathrm{CO}_{2}$ and volatile acids, was biased towards the production of lactic acid almost exclusively under the influence of the two inhibitors; the amount of lactic acid formed to the glucose degraded was 30 per cent for the control, 9o par cent for acrylate and 9I per cent for chlortetracycline. But the rate at which lactic acid was formed was increased by acrylate, $2.77 \mathrm{~g}$ lactic acid per $\mathrm{g}$ dry weight of microorganisms, compared with $0.82 \mathrm{~g}$ for the control. It was reduced by chlortetracycline, $0.73 \mathrm{~g}$.

4. These results could explain, at least in part, the increase which these two substances cause in growth of piglets, acting through improvement of utilisation of protein and of energy of the ration.

\section{RÉFÉRENCES BIBLIOGRAPHIQUES}

BARKer S. B., Summerson W. H., 194I. The colorimetric determination of lactic acid in biological material. J. Biol. Chem., 138, 535.

Bergey's. Manual of determinative bacteriology. Six ed. I948. The Williams and Wilkins Co. Baltimore. Conway E. J., I957. Microdiffusion analysis and volumetric error. Crosby-Lockwood London $4^{\text {th }} \mathrm{ed}$.

DANG H. C., VISEK W. J., I960. Effect of urease injection on body weights of growing rats and chicks. Proc. Soc. Exper. Biol. Med., 105, I64-167.

Delort-Laval J., Charlete-Léry G., Zelter S. Z., ig63. Efficacité de quelques protides alimentaires chez le porc. IV. Données complémentaires sur l'action de l'auréomycine sur le métabolisme azoté. Ann. Biol. ann. Bioch. Biophys., 3, 369-380.

Dickinson A., ig6r. Travaux de la Commission des antibiotiques du C.N.E.R.N.A Ann. Nutr. alim., $15,84-90$.

Dubos R. J., Schaedler R. W., 1960. The effect of the intestinal flora on the growth rate of mice, and on their susceptibility to experimental infections. J. Exper. Med., 111, 407-4I8.

FRANÇOIS A. C., 1962. Mode of action of antibiotics on growth. World Review of nutrition and dietelics, Pitman Medical Publishing Co.

Françors A. C., Michel M. C., 1963. Flore du tractus digestif et nutrition de l'hôte. Rôle des antibiotiques. Symposium sur la digestion des protéines. Glasgow (sous presse).

Françors A. C., MrCher M., I96o. Effets métaboliques de certaines flores digestives, conséquences nutritionnelles. Cah. Coll. Hôp., n ${ }^{0}$ I $2,949-955$.

Gale E. F., 1946. The bacterial aminoacid decarboxylases. Adv. Enzymol., 6, I-32.

György P., I957. Nutrition and intestinal flora in man. Ann. nutr. alim., 11, 189-206.

KREBS H. A., I954. The tricarboxylic cycle. Chemical pathacays of metabolism. Vol. I. Academic Presse New York.

Larson N. L., Hill E. G., 1960. Amine formation and metabolic activity of microorganisms in the ileum of young swine fed chlortetracycline. J. Bact.eriol., 80, I88-I92.

LEWIS T. R., EMERY R. S., 1962. Relative deamination rates of aminoacids by rumen microorganisms. J. Dairy Sci., 45, 765-768.

MASKA J., rg6o. The mecanism of effect of antibiotics in animal nutrition. Part. II. Zivocisna Vyroba., $33,35^{\mathrm{I}-362 \text {. }}$

Mendel B., Kemp A., Myers D. R., I954. A colorimetric micromethod for the determination of glucose. J. Biol. Chem., 56, 644 .

Melnikowicz J., Johansson K. R., i955. Formation of amines by intestinal microorganisms and the influence of tetracycline. J. Exp. Med., 101, 507-517.

Micher M. C., ig6r $a$. Application de la méthode de Nessler au microdosage de l'azote alpha-aminé en milieu biologique. Ann. Biol. anim. Bioch. Biophys., 1, 449-456.

Michel M. C., I961 $c$. Métabolisme de la flore intestinale du porc. Influence de la chlortétracycline sur le catabolisme du glucose. Ann. Biol. anim. Bioch. Biophys., 1, 213-221.

Michel M. C., 196г $d$. Activité métabolique de la flore totale isolée de l'intestin du porc. Rôle des différentes espèces microbiennes. Ann. Biol. anim. Bioch. Biophys., 1, I6-28.

Michel M. C., Jouandet G., Salmon-Legagneur E., Aumaitre A., Françols A. C., ig64. Influence de l'acrylate de sodium sur la croissance du porcelet. Ann. Zootech. 13, 341-350.

Raibaud P., I958. Travaux de la Commission des antibiotiques, du C. N. E. R. N. A. Ann. Nutr. alim., 12, I 35-105.

Raibaud P., r96r. Travaux de la Commission des antibiotiques, du C. N. E. R. N. A., Ann. Nutr. alim., 15, 79-83. 
Sieberti J. M. N., rg6o. Acrylic acid, an "antibiotic " principle in phacocytis blooms in antartic waters. Science, 132, 676-667.

SIEBURTh J. M. N., rg6r. Antibiotic properties of acrylic acid, a factor in the gastrointestinal antibiosis of polar marine animals. J. Bact, 82, 72-79.

VISEK W. J., 1962. Studies on urea hydrolysis in birds and mammals. Am. J. Vet. Res., 23, 569-574. White-Stevens R. II., Pevsack J. M., Stokstad E. L. R., Sieburth J. M. N., ig6z. The effect of acrylic acid salts on growth of chicks. Poult. Sci., 41, I909-I9r 5.

Wuruser R., I935. L'électroactivilé dans la chimie de cellules. Hermann et Cle. Paris.

Zelter S.Z., 1961. Travaux de la Commission des antibiotiques du C. N. E. R. N. A., Ann. Nuir. alim. 15, 105-IIO. 